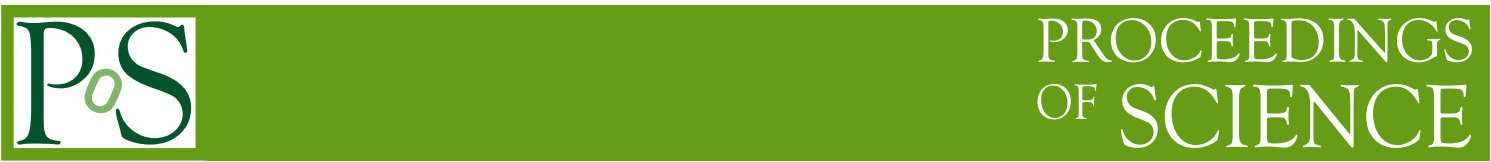

\title{
Bottomonium Studies at BaBar
}

\author{
Veronique Ziegler ${ }^{* \dagger}$ \\ SLAC National Accelerator Laboratory \\ E-mail: vziegler@slac.stanford.edu
}

Selected studies in bottomonium physics carried out by the BaBar experiment at the SLAC PEP-II $e^{+} e^{-}$collider are presented. They include bottomonium decays to open charm, and the search for the $h_{b}(1 P)$ and the discovery of $\eta_{b}(1 S)$.

European Physical Society Europhysics Conference on High Energy Physics, EPS-HEP 2009,

July 16 - 222009

Krakow, Poland

* Speaker.

${ }^{\dagger}$ On behalf of the BaBar Collaboration. 


\section{A brief overview of the bottomonium system}

Bottomonium bound states are the heaviest of the $q \bar{q}$ bound states. They have Parity $(-1)^{L+1}$ and Charge Conjugation $(-1)^{L+S}$ where $S$ is total spin and $L$ the total angular momentum of the $q \bar{q}$ system. The $b \bar{b}$ states below the $\Upsilon(3 S)$ that have not yet been discovered include two $S$-wave states $\left(\eta_{b}(2 S, 3 S)\right)$, two $P$-wave states $\left(h_{b}(1 P, 2 P)\right)$, four $D$-waves and possibly four $F$-wave states. The $\Upsilon(n S)$ resonances undergo hadronic transitions via $\pi^{0}, \eta, \omega$ or di-pion emission, electric dipole transitions or magnetic dipole transitions. The lowest mass bottomonium state, the $\eta_{b}(1 S)$, was discovered in $\Upsilon(3 S) \rightarrow \gamma \eta_{b}$ decay [1], and subsequently confirmed in $\Upsilon(2 S) \rightarrow \gamma \eta_{b}$ decay [2], in the BaBar experiment. Electromagnetic transitions between the energy levels can be calculated in the quark model and are an important tool in understanding the bottomonium internal structure. In particular, the measurement of the hyperfine mass splittings between the triplet and singlet states in quarkonium systems is of key importance in understanding the role of spin-spin interactions in quarkonium models and in testing QCD calculations.

\section{The $\eta_{b}(1 S)$ state}

One of the expected production mechanisms of the $\eta_{b}\left(n^{\prime} S\right)$ is by (magnetic) $M 1$ transition from the $\Upsilon(n S)\left(n^{\prime} \leq n\right)$ states. Since the decays of the $\eta_{b}$ are not known, the BaBar search strategy consisted of extracting the signal for $\Upsilon(3 S) \rightarrow \gamma \eta_{b}$ from a fit to the inclusive photon energy spectrum in the CM frame; the analysis made use of 109 million $\Upsilon(3 S)$ decays. The monochromatic photon from the decay appears as a peak around $E_{\gamma}=900 \mathrm{MeV}$ on top of a smooth non-peaking background from continuum ( $e^{+} e^{-} \rightarrow q \bar{q}$ with $\left.q=u, d, s, c\right)$ events and bottomonium decays. Sequential radiative decays $\Upsilon(3 S) \rightarrow \gamma \chi_{b J}(2 P), \chi_{b J}(2 P) \rightarrow \gamma \Upsilon(1 S)$, where $J=0,1,2$, produce a broad peak centered at $760 \mathrm{MeV}$ due to photons from decay of the $\chi_{b J}(2 P)$ states. This $\chi_{b J}(2 P)$ photon peak is well-separated from the signal region around $E_{\gamma}=900 \mathrm{MeV}$. A second process, which leads to a peak near $860 \mathrm{MeV}$ in the photon energy spectrum, is the production of the $\Upsilon(1 S)$ via initial state radiation (ISR) $e^{+} e^{-} \rightarrow \gamma_{I S R} \Upsilon(1 S)$. The $\eta_{b}$ signal is extracted from a fit to the net $E_{\gamma}$ spectrum in the region $0.5<E_{\gamma}<1.1 \mathrm{GeV}$ using a function consisting of a non-peaking background, $\chi_{b J}(2 P) \rightarrow \gamma \Upsilon(1 S)$, ISR and $\eta_{b}$ signal contributions (Fig. 1). Under the bottomonium interpretation, this is the first evidence for the $\eta_{b}$ bottomonium state, the pseudoscalar partner of the $\Upsilon(1 S)$. The $\mathrm{BaBar}$ measurement of the $\eta_{b}$ mass is $9388.9_{-2.3}^{+3.1} \pm 2.7 \mathrm{MeV} / c^{2}$, which corresponds to a mass splitting between the $\Upsilon(1 S)$ and the $\eta_{b}$ of $71.4_{-3.1}^{+2.3} \pm 2.7 \mathrm{MeV} / c^{2}$. The estimated branching fraction of the decay $\Upsilon(3 S) \rightarrow \gamma \eta_{b}$ is found to be $(4.8 \pm 0.5 \pm 1.2) \times 10^{-4}$.

This observation was then confirmed from a search for the $\eta_{b}(1 S)$ in the radiative decay of the $\Upsilon(2 S)$ resonance using a sample of 91.6 million $\Upsilon(2 S)$ events.

The ratio of the radiative production rates for this state from the $\Upsilon(2 S, 3 S)$ resonances is consistent with that expected for the $\eta_{b}$. Under this interpretation, the mass of the $\eta_{b}$ is $9394.2_{-4.9}^{+4.8} \pm 2.0$ $\mathrm{MeV} / \mathrm{c}^{2}$, which corresponds to a mass splitting between the $\Upsilon(1 S)$ and the $\eta_{b}$ of $66.1_{-4.8}^{+4.9} \pm 2.0$ $\mathrm{MeV} / \mathrm{c}^{2}$, consistent with the value from the $\Upsilon(3 S)$ analysis. The average of the two results is $M\left(\eta_{b}\right)=9390.8 \pm 3.2 \mathrm{MeV} / c^{2}$. This value of the $\eta_{b}$ mass is consistent with a recent unquenched lattice prediction [3], but is more than two standard deviations away from the mass predicted by approaches based on perturbative QCD [4]. 

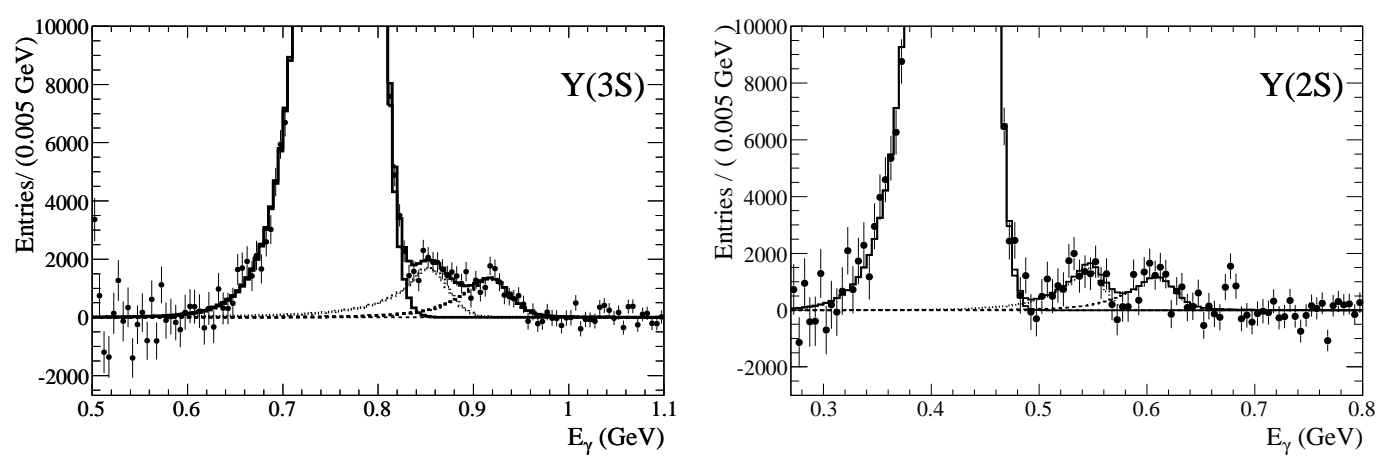

Figure 1: The inclusive $\Upsilon(3 S, 2 S) \rightarrow \gamma \eta_{b}(1 S)$ photon spectra after subtracting the non-peaking background contributions. In each plot, the peaks from low to high energy correspond to the $\chi_{b J} \rightarrow \gamma \Upsilon(1 S)$, ISR and $\eta_{b}$ signal contributions, respectively.

\section{The search for the $h_{b}(1 P)$ state}

The $h_{b}(n P)$ is the $b \bar{b}$ spin-singlet partner of the P-wave $\chi_{b}(n P)$ states. QCD-based potential models used in predicting the masses of quarkonium states contain a Coulombic potential $(\sim 1 / r)$ and a confining potential $(\sim r)$ assumed to be scalar and expected to carry no spin dependence. Hence under this assumption, there should be hyperfine (spin-spin interaction) splitting, $\Delta M_{h f}=$ $M\left({ }^{3} S_{1}\right)-M\left({ }^{3} S_{0}\right)$, only for $L=0$ states, and the hyperfine splitting, $\Delta M_{h f}=M\left({ }^{3} P_{J}\right)-M\left({ }^{3} P_{0}\right)$ for $L=1$ states should be zero. Thus the mass of the spin-singlet should be equal to the spin-averaged mass of the ${ }^{3} P_{J}$ states, resulting in an expected $h_{b}(1 P)$ mass at the center of gravity of the $\chi_{b J}(1 P)$ states (i.e. $\sim 9900 \mathrm{MeV} / \mathrm{c}^{2}$ ). Higher-order corrections are expected to provide no more than a few $\mathrm{MeV}$ deviation from this result; a larger deviation might be indicative of a vector component in the confinement potential [5]. Note that the hyperfine splitting between the $h_{c}(1 P)$ state discovered by the CLEO experiment, and the centroid of the $\chi_{c J}(1 P)$ states was found to be $+1 \pm 0.6 \pm 0.4$ $\mathrm{MeV}[6]$.

The expected decays from the $\Upsilon(3 S)$ to the $h_{b}(1 P)$ are the isospin-violating $\Upsilon(3 S) \rightarrow \pi^{0} h_{b}(1 P)$ and the di-pion $\Upsilon(3 S) \rightarrow \pi^{+, 0} \pi^{-, 0} h_{b}(1 P)$ transitions. Each transition is expected to have a very small branching fraction (at most $10^{-3}$ ). The total width of the $h_{b}(1 P)$ is expected to be of the order of $100 \mathrm{keV}$, and a branching fraction to $\gamma \eta_{b}(1 S)$ larger than $40 \%$ is expected [7].

A search for the $\Upsilon(3 S) \rightarrow \pi^{0} h_{b}(1 P)$ transition and consists of looking for a signal in the vicinity of $9.9 \mathrm{GeV} / \mathrm{c}^{2}$ in the inclusive missing mass distribution against a $\pi^{0}$. This corresponds to a $\pi^{0}$ momentum of $425 \mathrm{MeV} / \mathrm{c}$ in the $e^{+} e^{-} \mathrm{CM}$ frame. This search is ongoing.

\section{Measurement of the decay $\Upsilon(1 S) \rightarrow D^{* \pm} X$}

The bound states of heavy quarks provide a powerful testing ground for Quantum Chromodynamics (QCD). Hadronic decays of the narrow quarkonia states below the threshold for open flavor production, are dominated by couplings to gluons and the fragmentation process into light hadrons. Little is known about the final states resulting from the decay of bottomonia. In particular, scarcely 


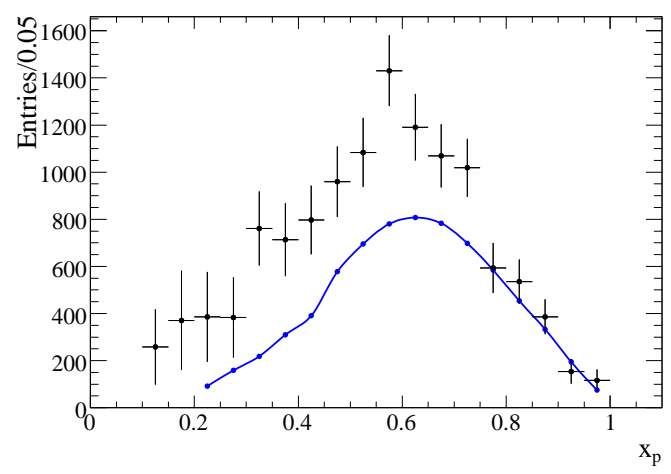

Figure 2: The $D^{*+}$ signal yield as a function of $x_{p}$. The solid curve represents the expected contribution from the virtual photon process [9].

any experimental information exists on the decay of bottomonium states to open charm. The inclusive process $\Upsilon(1 S) \rightarrow D^{* \pm} X$ was studied using data recorded by the BaBar collaboration at the $\Upsilon(2 S)$ resonance, where the $\Upsilon(1 S)$ sample was obtained by means of the $\pi^{+} \pi^{-}$transition from the $\Upsilon(2 S)$ [8]. The subsequent decay $\Upsilon(1 S) \rightarrow D^{* \pm} X$ can proceed through the QED virtual photon annihilation process, $\Upsilon(1 S) \rightarrow \gamma^{*} \rightarrow c \bar{c}$, followed by the hadronization of the $c \bar{c}$ system. The expected decay rate and the $D^{* \pm}$ momentum spectrum from this process can be accurately estimated from the measured properties of the $\Upsilon(1 S)$ decays and the charm fragmentation function at $\sqrt{s} \sim 10 \mathrm{GeV}$. Other QCD processes, such as gluon splitting or the annihilation of the $b \bar{b}$ system in an octet state, have also been suggested as major contributors to this decay process. Measurements of the $D^{* \pm}$ yield and its momentum spectrum can help test the predictions of the proposed QCD mechanisms, and possibly reveal the presence of new physics processes with exotic couplings to heavy quarks. Fig. 2 shows the expected scaled momentum $x_{p}=\frac{p_{D^{* \pm}}}{p_{\max }}$ distribution for $D^{* \pm}$ production from the QED virtual photon annihilation process, $\Upsilon(1 S) \rightarrow \gamma^{*} \rightarrow c \bar{c}$, where the shape is obtained from the measured $D^{* \pm}$ fragmentation function at $\sqrt{s}=10.5 \mathrm{GeV}$ [9]. The measured branching fraction exceeds the expected rate from the QED virtual photon process by $(1.07 \pm 0.28) \%$ (including the systematic error) which corresponds to 3.8 standard deviations. While the measured $x_{p}$ spectrum agrees in shape with that of the virtual photon process for $x_{p}>0.75$, there is a significant excess for $x_{p}<0.75$. The probability, estimated from a binned $\chi^{2}$ test, that the measured spectrum is consistent with the expected distribution from the virtual photon is $1.3 \times 10^{-6}$.

\section{Conclusions}

The large BaBar data samples collected at the $\Upsilon(3 S)$ and $\Upsilon(2 S)$ resonances allow measurements of the known bottomonium transitions with very high precision, and may, possibly lead to first observations of many of the missing states, thus providing useful checks of QCD predictions for the $b \bar{b}$ system. The $\eta_{b}$ observation is only the first of these, and the search for the $h_{b}$ and others is ongoing. 


\section{References}

[1] B. Aubert et al. [BaBar Collaboration], Phys. Rev. Lett. 101, 071801 (2008); 102, 029901(E) (2009).

[2] B. Aubert et al. [BaBar Collaboration], arXiv:0903.1124

[3] A. Gray et al. [HPQCD and UKQCD Collaborations], Phys. Rev. D 72, 094507 (2005). T. Burch and C. Ehmann, Nucl. Phys. A797, 33(2007).

T.-W. Chiu et al. [TWQCD Collaboration] Phys. Lett. B651, 171 (2007).

[4] B. A. Kniehl et al., Phys. Rev. Lett. 92, 242001 (2004).

S. Recksiegel and Y. Sumino, Phys. Lett. B578, 369 (2004).

[5] S. Godfrey and J. L. Rosner, Phys. Rev. D66, 014012 (2002).

[6] J. L. Rosner et al. [CLEO Collaboration], arXiv: hep-ex/0505073v2

[7] J.L. Rosner et al., Phys. Rev. Lett. 95, 102003 (2005).

[8] B. Aubert et al. [BaBar Collaboration], arXiv:0911.2024

[9] M. Artuso et al. [CLEO Collaboration], Phys. Rev. D 70, 112001 (2004). 